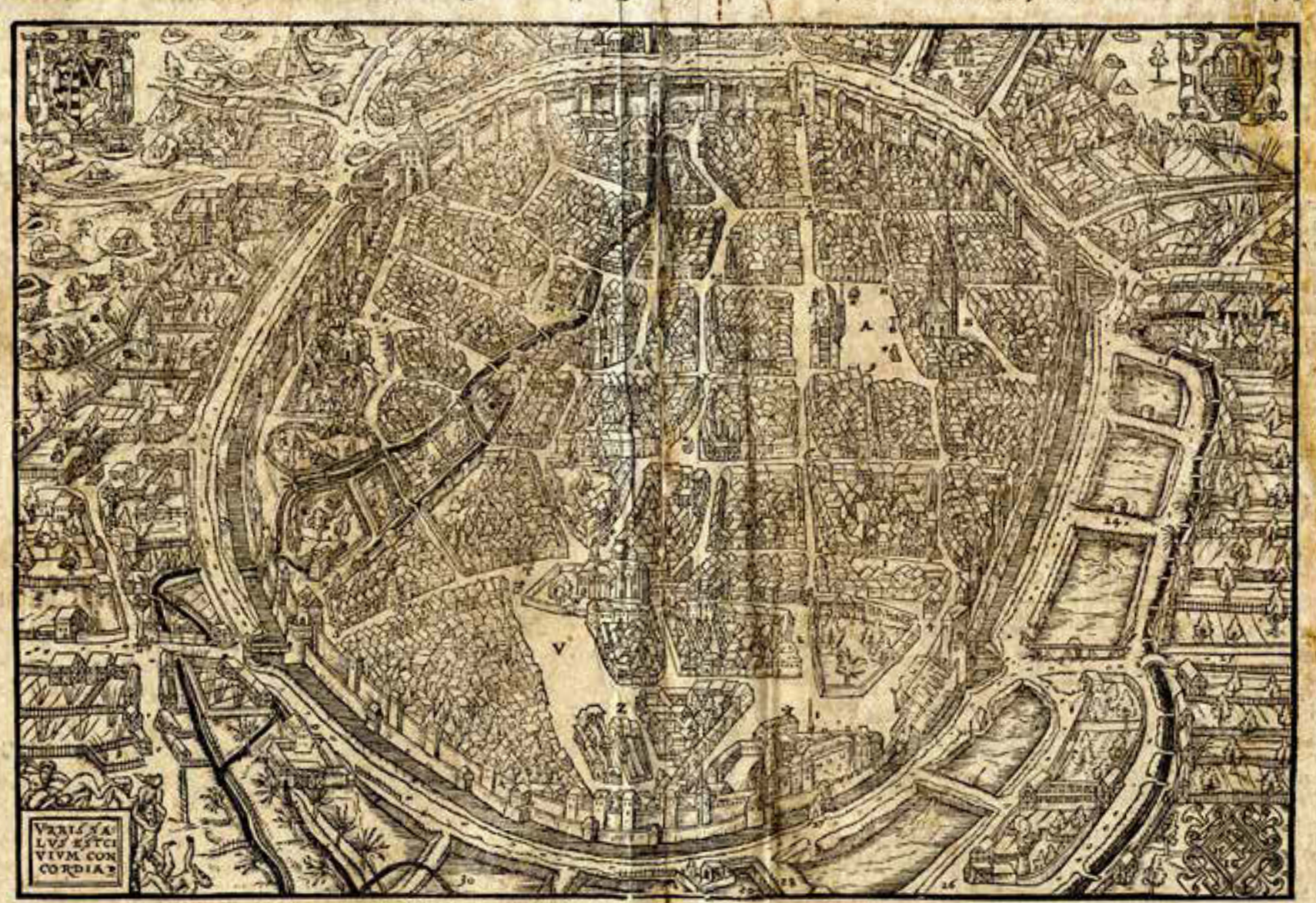

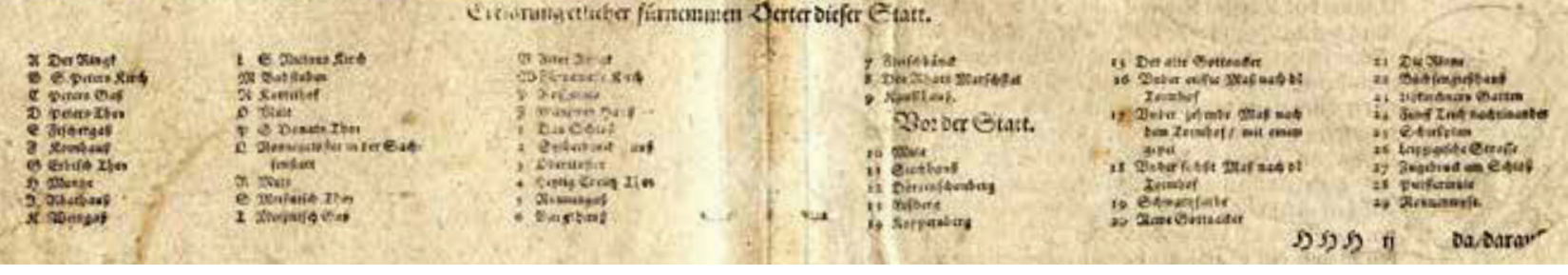

\title{
Die Reformation in Freiberg
}

\section{Karl-Hermann Kandler}

Vorgeschichte

Die Einführung der Reformation 1537 im Herrschaftsgebiet Herzog Heinrichs des Frommen (1473-1541), jüngerer Bruder des regierenden Herzogs Georg des Bärtigen (1471-1539), hat Modellcharakter für die zwei Jahre später erfolgte Einführung der Reformation im albertinischen Sachsen. Während Herzog Georg durch die von ihm unterstützte Disputation Luthers mit Eck in Leipzig zu einem Gegner Luthers geworden war, weil dieser sich positiv über Jan Hus (1369-1415) geäußert hatte, ${ }^{1}$ war Heinrich allmählich, vor allem durch den Einfluss seiner Frau Katharina (1487-1561), einer mecklenburgischen Herzogstochter, für die Reformation gewonnen worden.
Der Entscheidung Heinrichs für die Reformation hatte sich im Verlauf von zwanzig Jahren entwickelt. Der Freiberger Chronist Andreas Möller betont, es habe „viel Verhindernus gegeben/ und ist alles sehr schwer angangen“, bis es zur Einführung der Reformation kam. So hatte Heinrich 1523 drei Hoffräulein seiner Frau des Hofes verwiesen, weil bei ihnen Schriften Luthers gefunden worden waren; Luther schrieb ihnen deswegen einen Trostbrief. ${ }^{2}$ Freiberger Bürger wurden bestraft, weil sie sich nicht mehr an die kirchlichen Fastengebote hielten. ${ }^{3}$ Schon längst hatten viele Freiberger sich innerlich der Reformation zugewandt. Die Kritik an der Freiberger Geistlichkeit war immer lauter geworden. Der Freiberger Domherr Bernhard Hempel hatte zwei Jungen, die ihm Äpfel gestohlen
Ansicht von Freiberg, um 1600

1 Dazu M. Brecht: Martin Luther. Sein Weg zur Reformation, Stuttgart 1981, Lizenz Leipzig 1986, S. 285-332, besonders S. 304 f. und 346 Zum Ganzen: G. Wartenberg: Die Einwirkungen Luthers auf die reformatorische Bewegung im Freiberger Gebiet und die Herausbildung des evangelischen Kirchenwesens unter Herzog Heinrich von Sachsen, in: Herbergen der Christenheit, Bd. 12 (1981/82), Berlin 1982, S. 93-117; jetzt in ders.: Wittenberger Reformation und 
Herzog Heinrich der Fromme in der Grabkapelle des Freiburger Domes Foto: Karl-Hermann Kandler

territoriale Politik, Leipzig 2003, S. 121-146: K.-H. Kandler: Kirchengeschichte Freibergs 1162-1648, Beucha/Markkleeberg 2013.

2 A. Möller: Theatrum Freibergense Chronicum, Band I, Freybergk 1653, S. 231. Ein Sendbrief D. M. Luthers an die drei Hofjungfrauen ... vom 18. Juni 1523, Werke, Weimarer Ausgabe (WA), Briefe, Bd. III, S. 93 f., Nr. 625

3 Möller, Bd. I, S. 214

4 A.a.O., S. 208; Bd. II, S. 177 f,

A.a.O., Bd. I, S. $214 \mathrm{f}$

6 A.a.O., Bd. II, S. 170.

7 A.a.O., Bd., II, S. 179 f.; M. Luther: Deutung der zwei gräulichen Figuren, Papstesels zu Rom und Mönchkalbs zu Freiberg in Sachsen gefunden, WA, Bd. 11, S. 369-385.

8 Freiberger Urkundenbuch (künftig: FUB), Bd. I, Nr. 705-713, S. 477-485; Nr. 716, S. 496. Möller: A.a.O., Bd. II, S. 185, dazu M. Luther: Der durchlauchtigen Ursula, Herzogin von Münsterberg, „Christliche Ursach des Verlassens des Klosters zu Freiberg", Nachwort, WA, Bd. 26, S. 628-633.

9 Möller: A.a.O., Bd. I, S. 51; Bd. II, S. 131, 137. Dazu H. Petzold: Der Streit um die Freiberger „Butterbriefe“, in: Herbergen der Christenheit, Sonderband: Das Hochstift Meißen, Berlin 1973, S. 147-164.

10 Möller: A.a.O., Bd. I, S. 215 f.; Y. Hoffmann/U. Richter: Entstehung und Blüte der Stadt Freiberg. Halle/S. 2012, S. $631 \mathrm{f}$.

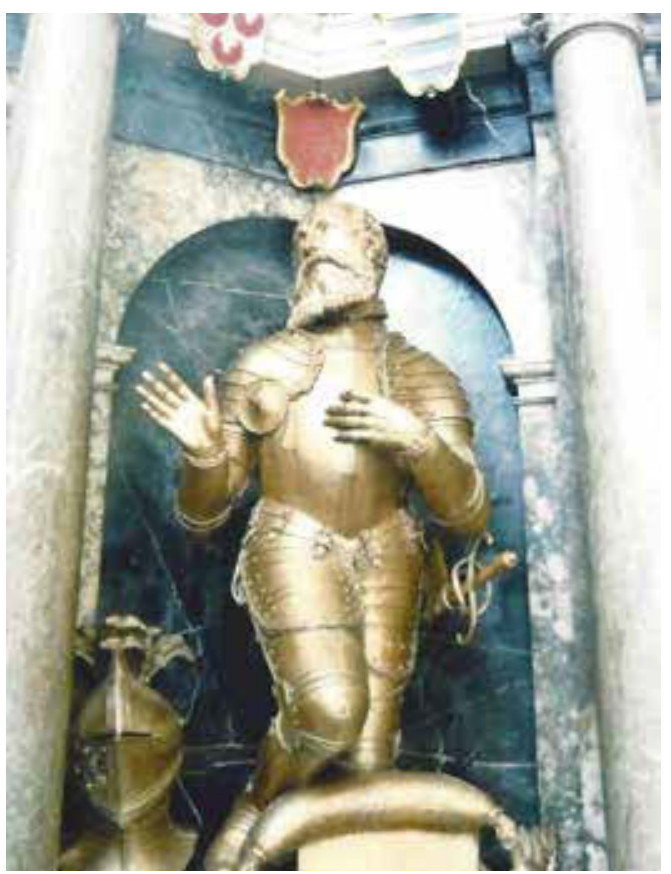

hatten, so geschlagen, dass einer von ihnen an den Folgen der Schläge verstarb. Manche Geistliche der Stadt wurden gefoppt, „denn der Päbstischen Geistlichen Betrug und Tauscherey dem gemeinen Volck schon allzubekant war“. Auch die Geschichte von der „schönen Polyxena“ erregte Unwillen dem Stiftskapitel gegenüber. Die Tochter des Domherrn Johann Gertewitz hatte ihren Mann Johann Beham erschlagen. Wegen Ehebruchs und Mords wurde sie hingerichtet. ${ }^{4}$

Sicher, es gab auch Positives über die Freiberger Pfarrer an den Stadtkirchen zu berichten, aber Heinrich fühlte sich veranlasst, ein Mandat an den Rat zu schicken, „darinnen befohlen wird/ daß sie der Gemeine wehren sollen/ damit die ThumHerren und Prediger zu S. Petri und Nicolai nicht schimpffieret/ sie auch und andere Priester und Münche auff der Gasse und in ihren eigenen Häusern und Klöstern/ wie denn Ihrer Gnad. durch glaubwürdigen Bericht fürkommen/ und sie solches mit gantz verdrießlichen Gemüthe vernehme/ nicht mehr belästiget würden“.

Zwei Franziskaner und ein Dominikaner (Georg Schumann) verließen ihre Klöster, auch der Kaplan an St. Nikolai Johann Böhm und der Stadtgeistliche Dominicus Beyer pflichteten „des Herrn Doctor Lutheri Göttlichen Lehre“ bei. Darum erließ der Herzog ein weiteres Mandat, dass man den Priestern die ihnen schuldigen Opfer und Zinsen „unabbrüchlich“ weiter reichen solle. Ebenso wurden die Fastengebote erneut bekräftigt. ${ }^{5}$ Doch schon längst hatten die Freiberger Fakten geschaffen. Bereits 1517 hatten sie Johann Tetzel, als er auch in Freiberg den Ablass vertreiben wollte, abgewiesen (,also gar/ daß nicht allein wenig Personen seiner geachtet/ sondern auch die Bergleute ihn zu beschimpffen sich unterstanden/ und verlauten lassen/ das gesamlete Ablaßgeld ihm gar abzunehmen“). ${ }^{6}$

Bekannt geworden ist auch die Geschichte vom „Mönchskalb“, einer Missgeburt, dessen Kopf mit etlicher Phantasie einem Mönchskopf geglichen haben soll. Nicht nur Luther hat darauf ein satirisches Gedicht verfasst, sondern auch die Freiberger Bergleute haben „ein besonder schimpfflich Lied davon erdichtet und dasselbe den Mönchen und Pfaffen zu spott und hohn lange zeit allhier gesungen".7

1528 floh die Nonne Herzogin Ursula von Münsterberg aus dem Freiberger Magdalenerinnenkloster - zusammen mit zwei weiteren Nonnen. Ein Jahr später entwichen erneut vier Nonnen aus dem Kloster. ${ }^{8}$

Bekannt geworden ist auch der Streit um die sogenannten Butterbriefe. Papst Innozenz VIII. hatte 1491 zugelassen, dass gegen die Zahlung eines Zwanzigstel Rheinischen Guldens die ganzen Fastenzeiten hindurch von den Freibergern Butter- und Milchspeisen gegessen werden durften. Das Geld, das dadurch einkam, sollte nach Abzug eines Viertels, das für den Bau der Peterskirche in Rom abzuführen war, dem Wiederaufbau des 1484 abgebrannten Domes zugutekommen. Dagegen haben die Dominikaner und Franziskaner „starck darwider protestiret“, „solches in Predigten hart geeifert" und den Dechanten mit seinen Domherren beschuldigt. 1496 wurden den Klöstern durch Papst Alexander VI. „ein ewiges stillschweigen aufferleget".?

All diese Begebenheiten zeigen, wie sehr es in Freiberg gärte. Viele wünschten sich die Reformation der Kirche. Sie scheuten sich nicht, zu reformatorischen Gottesdiensten in die nächsten Orte außerhalb des Herzogtums zu laufen, manche sogar bis nach Wittenberg. Vor allem aber begannen sie, „ihre Begierde öffentlichen für iedermans Augen zu erkennen gegeben“, indem sie über „ihre HaußThüren/ die lateinische Buchstaben V.D.M.I.AE. auch deutsch und außdrücklich/ Gottes Wort bleibet in Ewigkeit/ in Stein neben der Jahrzahl einhawen lassen“. Am Haus Petersstraße 46 ist heute noch eine große Tafel (Abendmahlstafel) zu sehen. Auf ihr stehen die Anfangsbuchstaben der Einsetzungsworte des Abendmahls und die Anfangsbuchstaben des Bekenntnisses: VDMIAE, datiert 1529. Ob die Tafel ein allgemeines Bekenntnis zur Reformation darstellt oder sich darauf bezieht, dass hier (angeblich) zum ersten Mal das Abendmahl unter beiderlei Gestalt gefeiert wurde, ist umstritten. Ebenso umstritten ist, ob sich in diesem Hause eine Hauskapelle befunden hat. ${ }^{10}$

Der Rat als auch der Herzog reagierten auf die 
Kritik der Freiberger Bevölkerung. Der Rat ordnete „krafft habender Privilegien und alter päbstlicher Indulgentz“ 1523 die Visitation sowohl des Dominikaner- als auch des Franziskanerklosters an. Auf Heinrichs Veranlassung wurden 1530 erneut beide Klöster visitiert. Vermutet wurden Unregelmäßigkeiten hinsichtlich der Bewahrung der in den Klöstern vorhandenen Kleinodien. ${ }^{11}$ Zur gleichen Zeit fingen aber auch Mönche aus beiden Klöstern an, reformatorisch zu predigen. Bereits 1524/25 hat der Franziskaner Laurentius Soranus in seiner Klosterkirche reformatorisch gepredigt. ${ }^{12}$ Der Dominikaner Georg Schumann wurde Hofprediger in der Schlosskirche. Der Stadtgeistliche Dominicus Beyer heiratete schon 1524. Diese Geistlichen haben „die Gemeine in ihren Fürhaben höchlichen gestärcket“. ${ }^{13}$ So kam es dazu, dass die Freiberger „sich erkühnet/ und bey ihren gnädigsten Herrn/ hochgeachteten Hertzog Heinrichen zu Sachsen/ welcher damals seine Residentz und Hoffhaltung in der Stadt gehabt/ unterthänigste Ansuchung gethan/ daß Ihre Fürstl. Gnad. vergönnen und zulassen wolte/ damit das reine Wort Gottes öffentlich und unverhindert möchte geprediget/ die heiligen Sacramenta nach Ordnung und Einsetzung des HERRN Christi distribuiret, und hingegen die eingerissenen Päbstischen MenschenSatzungen abgeschaffet werden ..... Doch dem „gemeinen Mann“ hat man dies anfänglich nicht gewähren wollen, auch die Domherren und die anderen Geistlichen haben dies mit Macht zu verhindern gesucht und dabei Rückhalt bei Herzog Georg gefunden. Denen, die die alte Kirchenordnung nicht mehr billigten, wurden harte Strafen angedroht. Dies geschah auf Befehl Herzog Georgs. Möller schreibt, dass „der löbliche Fürst Hertzog Heinrich (war) der reinen Lutherischen Lehre selbst wol gewogen/ lies auch hernach die gefangenen Bürger ohn alles entgeld wieder los“. Das ist das erste Anzeichen dafür, dass Heinrich sich seinem Bruder in dieser Angelegenheit widersetzte. Er entschloss sich, den Wünschen der Freiberger nach einer Reformation der Kirche zu folgen. Durch Heinrichs Frau, Katharina von Mecklenburg, war Heinrich immer stärker in den Umkreis der Reformation gezogen worden. 1531 weilte das Herzogpaar in Torgau am kurfürstlichen Hof. Wahrscheinlich kam es bei dieser Gelegenheit zu einer ersten Begegnung mit Luther. Jedenfalls nahm Heinrich an einem Gottesdienst teil, in dem Luther gegen das Fasten predigte. Heinrichs Ausrede, er sei schwerhörig, half ihm nicht. Katharina ließ ihm extra einen Stuhl der Kanzel gegenüber aufstellen. ${ }^{14}$ Wenn auch in den Stadtkirchen weiterhin die altkirchlichen
Messen gefeiert wurden, so erlaubte Heinrich, dass Georg Schumann im Dominikanerkloster „iederman/ der es begehrte/ das H. Abendmal unter beyderley gestalt heimlichen reichen" ließ. Dem sah Heinrich zu und „vergunte“ es. ${ }^{15}$ Es kam so weit, dass die Freiberger nicht mehr bereit waren, den Stadtpfarrern zu ihrem Unterhalt beizutragen. 1533 begann Valentin Beltzing, Pfarrer an St. Nikolai, „öffentlich (anfieng) das Evangelium aus der KirchenPostill Lutheri von Wort zu Wort in der NiclasKirchen zu predigen“. Er war damit der erste, der öffentlich „Gottes wahres Wort lauter und rein $\mathrm{zu}$ Freyberg verkündiget“ “. ${ }^{16}$

Luther ist zwar nie in Freiberg gewesen, aber er hatte gute Kontakte zu einigen Freibergern. Enge Beziehungen bestanden vor allem zu den Geschwistern Weller. Hieronymus Weller (1499-1572) war als Student in Wittenberg nach einer Predigt Luthers tief betroffen und hat sich daraufhin zum Theologiestudium entschieden. 1530 bis 1536 war er Famulus in Luthers Haus und hat dessen Sohn Hans unterrichtet. Luther hat den Freiberger Rat um einen Beitrag für die Unkosten gebeten, die Weller bei seiner Promotion entstanden waren, ,sonderlich weil er euer Stadtkind ist“. Er sei „ein treu, still und gelehrter Mann“, es sei gut, „dass Ihr Euch solchen Leuten zu helfen annehmet“. Alle drei Geschwister, Hieronymus, Matthias und Barbara Lißkirchen (geb. Weller) waren schwermütig veranlagt. Luther hat ihnen seelsorgerliche Briefe geschrieben. Hieronymus wurde später Theologieprofessor an der hiesigen Stadtschule und genoss hohes geistliches und wissenschaftliches Ansehen. Matthias war

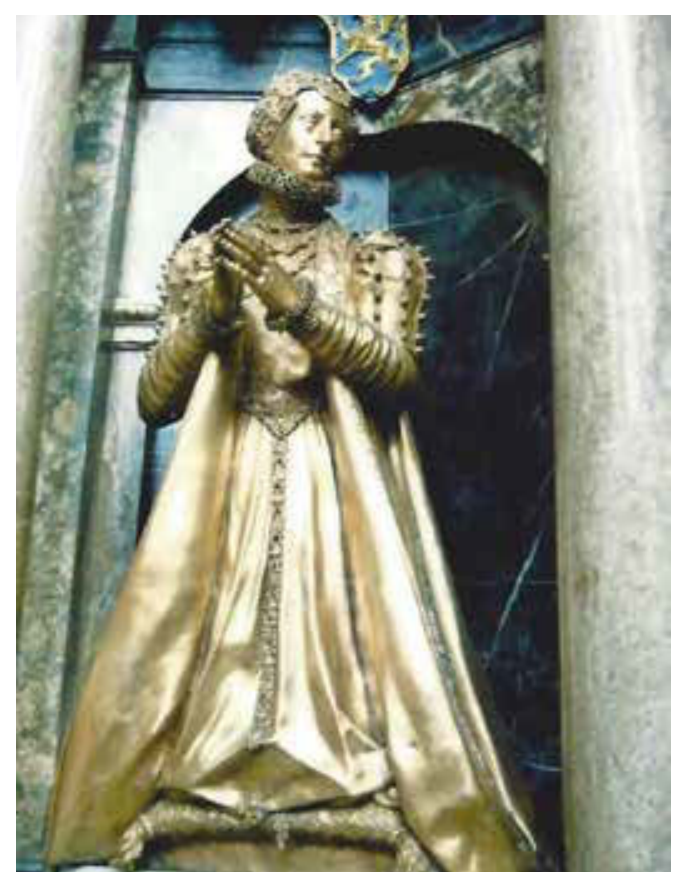

11 FUB, Bd. I, Nr. 547, S. 360; Nr. 556, S. 364; Möller: A.a.O., Bd. II, S. 181 f., 189 f. 12 FUB, Bd. I, Nr. 601, S. 393 ; F. Geß (Hrsg.): Akten und Briefe zur Kirchenpolitik Herzog Georgs von Sachsen, Bd. I: 1517-1524, Leipzig 1905, Nr. 763, S. 774-777. 13 Möller: A.a.O., Bd. I, S. 214. 14 A.a.O., Bd. I, S. 215 ff.; Georg Helts Briefwechsel, hrsg. von O. Clemen, Leipzig 1907, S. 76 (112).

15 Möller: A.a.O., Bd. I, S. 217. 16 A.a.O., Bd. I, S. 218.
Herzogin Katharina in der Grabkapelle im Freiberger Dom Foto: Karl-Hermann Kandler 
17 Dazu K.-H. Kandler: Luthers Beziehungen zur Freiberger Familie Weller auf Grund seiner Briefe, in: Mitteilungen des Freiberger Altertumsvereins, Heft 76, 1995 , S. 22-35. Darin Nachweise.

18 Luther: Werke, WA, Briefe, Bd. 7, S. 365-367; Möller: A.a.O., Bd. II, S. $200 \mathrm{f}$. Dazu K.-H. Kandler: Martin Luther und Freiberg, in: Sächsische Heimatblätter 43 (1997), Heft 6, S. 376-381.

19 Dazu ausführlicher Kandler: A.a.O.; P. Vetter: Lutherana I: Luthers Streit mit Herzog Heinrich von Sachsen, in: Neues Archiv für Sächsische Geschichte und Altertumskunde, Bd. 29, 1908, S. 82-94.

20 Möller: A.a.O.,Bd. I, S. 220 f.; Bd. II, S. 202.

21 A.a.O., Bd. I, S. 219 f.; J. Herrmann: Moritz von Sachsen. Landes-, Reichs und Friedensfürst, Beucha 2003, S. $25 \mathrm{f}$.

22 Sächsisches Staatsarchiv, Hauptstaatsarchiv Dresden (künftig: HStA), Loc. 9865/11, fol. 28a; vgl. H.-M. Kühn: Die Einziehung des geistlichen Gutes im albertinischen Sachsen 1539-1553. Graz/Köln 1966, S. 15.

23 HStA, Loc. 10001, Urk. 10850; vgl. H. Jadatz: Herzog Heinrich der Fromme als Förderer der Wittenberger Reformation und als evangelischer Landesherr, in: Herzog Heinrich der Fromme, hg. von Y. Hoffmann und U. Richter, Beucha 2007, S. 87 f.

Sandsteinrelief in der Domgasse 18 in Freiberg Foto: Karl-Hermann Kandler zunächst Hofmusiker, später Organist, Barbara Ehefrau eines Ratsherrn; sie hat sich auch mit theologischen Fragen an Luther gewandt. ${ }^{17}$ 1536 hat Luther an Lorenz Kastner geschrieben und ihn vor Winkelpredigten gewarnt, ging es doch darum, dass Gottes Wort öffentlich verkündigt werden muss. Wenn dies in Freiberg nicht möglich wäre, dann sollten die Leute lieber in die päpstliche Messe gehen oder in eine Kirche jenseits der Grenze des Herzogtums zum reformatorischen Gottesdienst. Vor allem untersagte Luther, das Abendmahl in den Häusern unter beiderlei Gestalt durch Laien heimlich $\mathrm{zu}$ empfangen. ${ }^{18}$ Andererseits hat sich Luther im selben Jahr um Matthes Lotther, einem Kartenmaler, gekümmert. Dieser war in Verruf geraten, er würde selbst in seinem Haus predigen und seinem Gesinde das Abendmahl reichen. Da er nicht ordiniert war, war dies, wie gesagt, nicht möglich. Matthes geriet in Verdacht, ein „Schwärmer“ zu sein. Herzog Heinrich ließ ihn verhaften. Angeblich war schon der Henker aus Dresden zu seiner Hinrichtung unterwegs. Matthes gelang die Flucht - zu Luther. Dieser hat ihn aufgenommen und das Gefühl gewonnen, ihm geschähe Unrecht. Wiederholt hat sich seinetwegen Luther an Heinrich gewandt und damit seinen Unwillen herauf beschworen - und das in den Wochen, in denen es um die Einführung der Reformation in Heinrichs Herrschaftsgebiet ging. So schlimm Schwärmerei auch für Luther war, so war doch das Schicksal dieses Mannes ihm so wichtig, dass er den Unwillen des Fürsten in Kauf nahm und im Grunde damit die Einführung der Reformation in Freiberg aufs Spiel setzte. ${ }^{19}$

\section{Die Einführung der Reformation in Freiberg}

Am 29. September 1536 gab Heinrich endlich die Erlaubnis zur Einführung der Reformation, das „freye öffentliche exercitium der reinen



Evangelischen Lehre/ inhalts der Augspurgischen Confession, dem Rathe und Bürgerschafft $\mathrm{zu}$ Freybergk gnädigst vergönnet“. ${ }^{20}$ Das war mutig, denn Heinrich war von seinem in Dresden regierenden Bruder zumindest finanziell abhängig und auf Zuwendungen angewiesen. Da Georgs Söhne alle früh verstorben waren und auch sein zuletzt noch lebender geisteskranker Sohn, den er noch verheiraten ließ, vor ihm kinderlos starb, versuchte er, Heinrich geradezu zu erpressen, damit dieser, falls er Georg überlebte, nicht die Reformation im Herzogtum einführte. Sogar von seiner privaten Erbfolge wollte er ihn ausschließen. Heinrich ließ sich aber nicht erpressen.

Heinrichs Entscheidung gingen einige Handlungen voraus, die als Anzeichen für eine Wende gedeutet werden müssen. 1535 nahm er Antonius von Schönberg (um 1480-1554), einen sich zur Reformation bekennenden Adligen, unter seine Räte. Heinrichs ältester Sohn Moritz wurde vom Dresdner Hof zurückgeholt und an den kurfürstlichen Hof nach Torgau bzw. Wittenberg gesandt. ${ }^{21}$ Als Heinrich das Exercitium erteilt hatte, ließ Georg daraufhin alle Einkünfte kirchlicher Einrichtungen, die sich in Heinrichs Herrschaftsgebiet befanden, sperren. ${ }^{22}$ Im Februar 1537 stellte Heinrich den Antrag auf Mitgliedschaft im Schmalkaldischen Bund, dem am 7. Juli 1537 entsprochen wurde. ${ }^{23}$

Auf Katharinas Wunsch hin sandte Luther einen unverheirateten Theologen - und zwar D. Jakob Schenk - nach Freiberg. Umstritten bleibt, ob dieser ordiniert war oder nicht. Jedenfalls hat er in Wittenberg promoviert. ${ }^{24}$ Zunächst predigte er in der Schlosskirche, aber am Neujahrstag 1537 wurde zum ersten Male öffentlich im Dom von ihm reformatorisch gepredigt und das heilige Abendmahl unter beiderlei Gestalt gefeiert und ausgeteilt. Das war das entscheidende Datum. Nun gab es kein Zurück mehr. Einige Monate gab es zwar auch noch in den Stadtkirchen die altgläubigen Messen, doch anlässlich der Visitation durch Wittenberger Theologen $\mathrm{zu}$ Pfingsten 1537 wurde die Reformation in der ganzen Stadt durchgesetzt. Schenk hatte „dem Volck die Augen vollends gäntzlich eröffnet“, so „wolte man ferner die Abergläubischen falschen Irrthume und Ceremonien in Kirchen und Klöstern nicht mehr dulden“. Den Mönchen und Stadtgeistlichen wurde durch Befehl des Herzogs geboten, „sich ihrer Winckelmessen und andern Götzendiensts zu enthalten“ Die Mönche und Geistlichen, die sich dieser Anordnung widersetzten und „sich nicht zur Augspurgischen Confession bekennen wollen“, wurden „ihrer Aempter und Bestallung entsetzet“ Dem gegenüber hat Schenk im Dom täglich zweimal 
gepredigt und sowohl sonntags als auch donnerstags das hl. Abendmahl unter beiderlei Gestalt gereicht. ${ }^{25}$ Es gab zwar noch einige Freiberger, die sich der Reformation verschlossen und sich dabei auf Melanchthon beriefen, er habe es in bestimmten Fällen für möglich gehalten, das Abendmahl auch weiterhin unter nur einer Gestalt zu feiern, ${ }^{26}$ doch wurde der Laienkelch verbindlich in Freiberg eingeführt. Widerstand gab es in einigen Klöstern, vor allem im Kloster der Magdalenerinnen. Mönche und Nonnen wurden vor die Entscheidung gestellt, sich entweder zur Reformation zu bekennen und aus dem Orden auszutreten oder die Stadt zu verlassen und in ein auswärtiges Kloster einzutreten. Aus dem Besitztum der Klöster sollte ihnen eine Rente als Abfindung gezahlt werden. Einen Zwang, sich der Reformation anzuschließen, scheint es jedoch nicht gegeben zu haben. Doch ganz freiwillig ging es bei den Mönchen nicht ab. Schenk schrieb am 8. Juni 1537: „Die schwarzen Pauler munch sind sehr halsstarrig“. ${ }^{27}$ Die Magdalenerinnen wurden unter Druck gesetzt. Sie wurden hinsichtlich der Visitationsartikel vernommen. Etliche weigerten sich, sich ihnen zu beugen. Sie wurden vor die Alternative gestellt, entweder zu bleiben und sich zur Augsburgischen Konfession zu bekennen oder das Kloster zu verlassen, wobei sie mit „notturfftiglichenn“ versehen wurden. Es wird deutlich, dass es hierbei nicht nur um eine Glaubensfrage ging, sondern auch um eine der Versorgung. Die war ihnen im Kloster zugesichert. Etliche von ihnen waren von ihrer (zumeist adligen) Familie ins Kloster geschickt worden, um darin versorgt zu werden. Ihre Familien, die dafür ja auch ein Eintrittsgeld hatten zahlen mussten, waren jetzt nicht mehr willens oder auch nicht in der Lage, sie zurückzunehmen. Das Kloster wurde schließlich säkularisiert, die verbliebenen Jungfrauen sollten sich „auskleiden“. Unter Kurfürst August wurde das Kloster - auch auf Drängen des Adels - eine Jungfrauenschule. Erst 1580 wurde das Kloster aufgehoben. ${ }^{28}$ Das Kollegiatstift wurde 1538 aufgelöst, aber hierzu, wie auch zu den Bettelordensklöstern, gibt es keine Urkunde mehr. Vom letzten Dechanten, Bernhard von Ragewitz, heißt es, er sei der Reformation „heftig zu wider gewesen“. Schließlich hat auch er die Augsburgische Konfession unterschrieben, wozu wohl auch die jährlichen 250 Gulden Abfindung beigetragen haben. ${ }^{29}$ Die Einkünfte der Klöster und des Kollegiatstiftes kamen dem schon 1531 eingerichteten „Gemeinen Kasten“ zugute, aus dem die „Kirchen- und Schuldiener“ bezahlt wurden. ${ }^{30}$ Ernstlichen Widerstand scheint es aber weder in der Bevölkerung noch unter Mönchen und Geistlichen gegeben zu haben, einige haben vielmehr geistliche Aufgaben im Sinne der Reformation übernommen.

Bitter beklagt hat sich dagegen der Abt des Klosters Altzella bei seinem Kollegen Abt Petrus in Schulpforta über die überhandnehmende Reformation in Freiberg. Er bezeichnete sie als Krankheit („morbus“), als Trübsal („tribulatio“) und spricht von einer Verschwörung der Lutheraner („Lutheranorum factio“). Er beschwerte sich darüber, dass Heinrich und sein ganzes Freiberger Volk Luther schon die Hand gereicht habe. Die Klöster seien verwüstet, der (altgläubige) Gottesdienst total untersagt. ${ }^{31}$

Die Einführung der Reformation in Freiberg muss als eine Glaubensentscheidung sowohl des Herzogs als auch der Bürgerschaft begriffen werden. Heinrich schrieb an seinen Bruder, dass er sich zur Augsburgischen Konfession bekenne, die „mit Claren gotlichenn schrifften ... dargethan wurdenn, die auch nochmals mit götlicher schrifft unwidderlegt blieben ist, die wir für Christlich, Evangelisch und Rechtschaffen halten“. ${ }^{32}$ Deshalb habe er der Einführung der Reformation in Freiberg zugestimmt.

Belastet wurde die Einführung der Reformation in Freiberg durch Jacob Schenk selbst. Er war ein sowohl kluger als auch eitler Mann, selbst wenn die Quellen sicher ein einseitiges, parteiisches Bild liefern. Jedenfalls zeigte er ein übersteigertes Selbstbewusstsein. Als der Rat forderte, auch in den anderen Stadtkirchen solle das heilige Abendmahl unter beiderlei Gestalt gefeiert werden, wehrte er sich dagegen. Er wollte, dass sich jedermann nach ihm richten solle und „satzte sich selbst zum obersten Bischoff“. Geeignete Männer ließ er wegziehen und holte seinen erst achtzehnjährigen Famulus Johann Fünfgülden als Prediger an den Dom. Er fiel nicht nur deswegen bei Luther in Ungnade, sondern vor allem wegen seiner Lehrabweichung. Mit Johann Agricola gehörte er zu den sogenannten Antinomern, die behaupteten, das alttestamentliche Gesetz solle nicht mehr in der Kirche gelten. Er soll wiederholt in seinen Predigten gesagt haben: „Glaube nur, und thue, was du wilt." Jedenfalls musste er sich nach einer deswegen erfolgten Visitation durch Justus Jonas, Georg Spalatin und Leonhard Beyer Ende Juni 1538 vor den Reformatoren verantworten und die Stadt verlassen. Spalatin hat in einer Predigt am 7. Juli $1538 \mathrm{im}$ Dom vor den ,irrigen fürgeben von Gesetze eiferig gewarnet“. Das Abendmahl wurde nun in allen Stadtkirchen schriftgemäß gefeiert und auf Deutsch gepredigt. Das bisherige Einkommen der Klöster und des Stiftskapitels wurde zur Erhaltung der Kirchen und Schulen dem geistlichen Kasten zugeführt. Dem Rat wurde

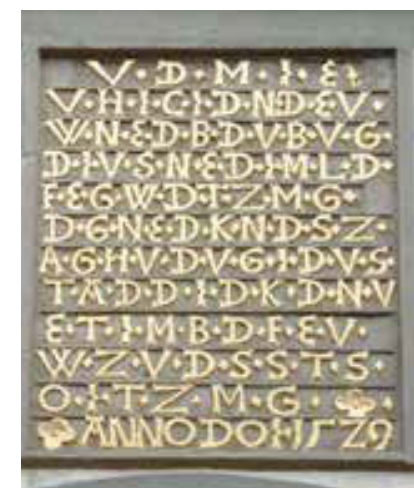

Abendmahlstafel am Haus

Petersstraße 46 in Freiberg

Foto: Karl-Hermann Kandler

24 Dazu K.-H. Kandler: D. Ja cob Schenk - Freibergs umstrittener Reformator, in: Mitteilungen des Freiberger Altertumsvereins, Heft 78, Freiberg 1997, S. 19-24 (Lit,). Bei Möller (a.a.O., Bd. I, S. 220) heißt es: „nach erlangten ordentlichen Beruff“, bei C. G. Wilisch: Kirchen-Historie der Stadt Freyberg, Leipzig 1737, S. 138: „nach erlangtem ordentlichen Beruff und Ordination“; aber S. 141: „ob er wol nicht geweihet“. Georgs Rat v. Carlowitz hat Schenk gedrängt: „,..., weil er noch nicht ordiniret, so sollte er sich den Bischoff von Meißen weihen lassen“. Das hat der Kurfürst abgelehnt. Im Wittenberger Ordinationsbuch ist eine Ordination Schenks nicht vermerkt (Auskunft der Wittenberger Superintendentur).

25 HStA, Akte „Das VisitationsWerck zu Freyberg belangende ao. 1537“, Loc. 9865 , Bl. 10-14; Möller: A.a.O., Bd. I, S. 220 f., 224; Bd. II, S. 202 f.; Kandler: A.a.O. (Anm. 18), S. 379.

26 Philipp Melanchthon Briefwechsel, hrsg. von H. Scheible, Bd. 2, Stuttgart 1978, Nr. 1903 f., 1922, 1930, 1932 f., 1941.

27 FUB, Bd. I, Nr. 562, S. 367.

28 FUB, Bd. I, Nr. 726-728, S. 504-508; Nr. 735, S. 511; Nr. 737, S. 521 f.; Nr. 741 f., S. $523 \mathrm{f}$.

29 FUB, Bd. I, Nr. 569, S. 371; Möller, Bd. I, S. 204 f.

30 HStA, Loc. 10594; FUB, Bd. I, Nr. 568 f., S. 370; Ephoralarchiv des Ev.-Luth. Kirchenbezirks Freiberg, Sign. 4362; Vgl. Möller: A.a.O., Bd. II, S. $190 \mathrm{ff}$. 
31 Möller: A.a.O., Bd. I, S. 221; Wilisch (Anm. 23), Bd. III, S. 198 f.; vgl. C. Ranacher: Die Zisterzienserabtei Altzelle in der Reformationszeit, in: Neues Archiv für sächsische Geschichte, Bd. 83, Neustadt/ Aisch 2012, S. 20 f.

32 HStA, Akte „Das VisitationsWerck zu Freyberg belangende de ao. 1537, Loc. 9865, Bl. 10-14, Zitat Bl. 11; Wilisch: A.a.O., Bd. I, S. 148, 155.

33 Wilisch: A.a.O (Anm. 24)., Bd. 1, S. 148 f.; Möller: A.a.O., Bd. II, S. 205 f.

34 Jadatz: A.a.O. (Anm. 23), S. 89 f.; Luther, Werke, WA, Briefe, Bd. 8, S. 179-183.

35 HStA, Loc. 9865/11, fol. 28a; Stadtarchiv Freiberg, Sign. KK No. 1, Akten II.I.26. Darin auch Heinrichs Kirchenvisitationsordnung Artikel des Patronatsrechts des Rates zu Freiberg 1537/ 38. Das Patronat an St. Jakobi (mit Nonnenkloster) fiel an den Landesherrn.

36 Wilisch: A.a.O, Bd. I, S. 149; K.-H. Kandler: Kirchengeschichte Freibergs, Bd. 1, S. 83-92.

37 HStA, Loc. 10594; Y. Hoffmann/U. Richter: Entstehung und Blüte der Stadt Freiberg, Halle/Saale 2012, S. 150; Kandler: A.a.O., S. 110112 .

38 HStA, Loc. 10289/21: fol. 16r f.: Reise- und Lebensbeschreibung Herzog Heinrichs von Sachsen von seinem Geheimschreiber Bernhard Freydinger. Auch abgedruckt bei U. Schirmer: Herzog Heinrich von Sachsen (14731541), in: Herzog Heinrich der Fromme (Anm. 23), S. 35 .

39 Möller: A.a.O., Bd. II, S. 212. 40 HStA, Loc. 10594/2, Bl. 22.

41 Stadtarchiv, Sign. KK 1, Nr. 1 (= FUB, Bd. I, Nr. 863, S. 625-634).

42 Luther: Werke, WA, Briefe, Bd. IX, Nr. 3638, S. 464.

43 Dazu A. Bartmuß: Die „Heinrichsagende“ und ihre Vorläufer. Zur Rezeption der Wittenberger Reformation im albertinischen Sachsen, Univ. Leipzig, Diplomarbeit 2003 (ungedruckt).

Autor

Prof. Dr.

Karl-Hermann Kandler Freiberg das Patronatsrecht, die Lehen und die Inspektion darüber übergeben. ${ }^{33}$

Belastend bei der Einführung der Reformation war auch der Schlossprediger Georg Karg (um 1512-1576), der spiritualistische Gedanken äußerte und an der Menschheit Jesu Christ zweifelte. Von Luther am 1. Januar 1538 verhört, wurde er vom Kurfürsten verhaftet, aber weil er sich belehren ließ, bald wieder freigelassen. ${ }^{34}$ Heinrich forderte in einem Schreiben vom 1. Oktober 1538, „gleichförmig ordnung und Reformation $\mathrm{zu}$ machen, vf das es darinnen in den Kirchen und Christlich gemeynden allenthalben eintrechtig und an einem enthe wie an dem andern gehaltenn werden möge“. Er befahl dafür eine erneute Visitation. ${ }^{35}$

Am 4. November 1538 wurde schließlich der sechzigjährige Nikolaus Hausmann als Superintendent eingeführt. Er war Sohn eines Freiberger Ratsherrn. Bei seiner Antrittspredigt erlitt er auf der sogenannten Tulpenkanzel einen Schlaganfall und starb noch am selben Tag. 1539 wurde dann Kaspar Zeuner am 27. Juli als Superintendent eingeführt. Neben ihm waren drei Geistliche am Dom tätig, dazu drei an St. Petri, drei an St. Nikolai, zwei an St. Jakobi und einer am Hospital. ${ }^{36}$ Die Stadtkirchen blieben als solche erhalten, die Kirchen der beiden Bettelorden wurden sehr bald abgerissen. Der Rat erbat sich Gebäude des Franziskanerklosters als Spital und erhielt sie auch. ${ }^{37}$

Als Herzog Georg am 17. April 1539 verstarb, übernahm Heinrich die Regierung des gesamten Herzogtums in Dresden. Von seinem Geheimschreiber Bernhard Freydinger liegt darüber ein Bericht vor, in dem es heißt: „Es kam aber Herzog Heinrich fast spät in Dresden ein, also daß man mit Fackeln leuchten mußte. In der Stadt waren Trauer und Freude durcheinander gemengt, was der alten Religion war, also Mönche, Pfaffen und ihr Anhang, waren betrübt. Der gemeine Mann lobte Gott, und wurden viele Gewaltige alsbald bekehrt, welche zuvor geschworen hatten, ehe sie denn lutherisch werden wollten, wollte sie ehe aus dem Lande ziehen."38 Heinrich nahm seinen Hofprediger Paul Lindenau mit nach Dresden. Bevor er 1541 starb, verfügte er, er wolle im Freiberger Dom begraben werden: „Er hette die Freyberger in aller Trew und Gehorsam gegen Gott und ihm befunden, drümb wolte er auch bey denselben ruhen und schlaffen. ${ }^{39}$

Um die Reformation in Freiberg zu befestigen, sind in den ersten Jahren nach ihrer Einführung jährlich Visitationen durchgeführt worden. Einige Geistliche, die zunächst einen Pfarrdienst übernommen hatten, wurden abgesetzt, da sie sich nicht bewährt hätten. Dagegen wurde ein ehemaliger Mönch für den Katechismusunterricht bestellt. Auf Anfrage der Visitatoren antwortete der Rat, es gäbe genügend Geistliche in der Stadt, die Gemeinden wollten mehr Gesang im Gottesdienst haben, auch solle vom Katechismus und vom Gebrauch des hochwürdigen Sakraments mehr gepredigt werden, wozu sich die Geistlichen auch bereitfanden. ${ }^{40}$

1542 wurden die geistlichen Güter aufgezeichnet und festgelegt, was darauf zur Bezahlung der Gehälter der Geistlichen, Lehrer und als Rente für alte Domherren und ehemalige Freiberger Ordensangehörige zu verwenden sei. Die Kleinodien wurden zum großen Teil eingeschmolzen oder verkauft, nur weniges blieb erhalten. ${ }^{41}$

\section{Ausblick und Resümee}

Auch nach Einführung der Reformation bestanden rege Beziehungen nach Wittenberg. 1543 wandte sich Zeuner an Luther hinsichtlich verschiedener Gottesdienstordnungen in beiden sächsischen Territorien. Luther beruhigte Zeuner: Bestünde Übereinstimmung in der Lehre, so sei eine solche bei den Zeremonien nicht nötig. Hieronymus Weller fragte, ob die das Abendmahl austeilenden Diakone verpflichtet seien, es selbst zu empfangen. Luther wollte diesbezüglich die evangelische Freiheit gewahrt wissen. Dagegen lehnte er es Weller gegenüber strikt ab, die im Zusammenhang mit der Einführung der Reformation geschlossenen Bordelle wieder zu öffnen. ${ }^{42}$ Wie in ganz Sachsen war die Zeit nach der Reformation von Auseinandersetzungen um die rechte Lehre geprägt. Darum hat Zeuner versucht, diese durch Visitationen zu klären und die auf Anordnung Heinrichs von den Wittenberger Reformatoren erstellte neue Gottesdienstordnung (sog. Heinrichsagende) einzuführen. ${ }^{43}$ Die vordringlichste Aufgabe war es, die Reformation zu konsolidieren. Mit wenigen Ausnahmen scheint die Einführung der Reformation reibungslos verlaufen zu sein, zumindest geben die Quellen nichts anderes her, auch wenn man berücksichtigen muss, dass diese ganz offensichtlich nicht vorurteilsfrei die Ereignisse wiedergeben.

Kirchengeschichtlich gesehen, hat die Bedeutung Freibergs mit der Einführung der Reformation im Herzogtum den Höhepunkt überschritten. Künftig spielte Freiberg nur noch eine übergeordnete Rolle als Bestattungsort der Wettiner im Dom (bis zur Konversion Kurfürst Friedrich Augusts I. zur römisch-katholischen Kirche). Es bleibt aber Tatsache, dass die Einführung der Reformation 1537 in Freiberg Modellcharakter für deren Einführung im ganzen Herzogtum nach der Übernahme der Regierung im Herzogtum durch Heinrich hatte. 